\title{
AN INFINITE-DIMENSIONAL SCHOENFLIES THEOREM
}

\author{
BY \\ D. E. SANDERSON
}

Although important high-dimensional cases of the polyhedral Schoenflies theorem are yet unsettled (see [4] and [11]), there has been considerable success in the past ten years in generalizing the theorem, proved by Schoenflies in 1906, to the effect that every one-dimensional sphere embedded in the plane is tame. Of course Alexander's horned sphere and others show the necessity of a further condition to obtain a generalization to embeddings of $S^{n}$ in $E^{n+1}$ for $n>1$. Alexander proved tameness in the combinatorial and differential categories for $n=2$, M. H. A. Newman extended the result to include all finite dimensions in the combinatorial category [10] and Barry Mazur accomplished the same for the differential category [7]. In the topological category, Marston Morse showed that Mazur's result implies tameness of semilocally tame (bicollared) $n$-spheres in $E^{n+1}$ [9] and at the same time Morton Brown gave an elegant proof independent of Mazur's result [3].

As might be expected, in infinite-dimensional normed linear spaces even the counterpart of the Jordan curve theorem does not hold. However, it is shown in this paper that with the bicollared condition one does get Jordan separation. In fact tameness is proved by a modification of Brown's technique. Hopefully this infinite-dimensional Schoenflies theorem will be as useful in infinite-dimensional topology as has been the case in finite dimensions. To give substance to this hope a number of applications are given including an annulus theorem, a contribution to the classification of convex sets and partial answers to questions raised by $\mathbf{R}$. $\mathbf{H}$. Bing and R. D. Anderson [2].

1.0 Definitions. Unless otherwise stated, $X$ denotes a topological space and $E$ (with or without subscripts or superscripts) an infinite-dimensional normed linear space with the norm topology. Let $B_{t}=\{x \in E|| x \mid \leqq t\}$ and $S_{t}=\{x \in E|| x \mid=t\}$ for $t>0$. If $A \subset X, \mathrm{Bd} A$ denotes the point-set boundary of $A$ and ${ }^{\circ} A$ its interior. If $h:\left(B_{2}, S_{2}\right) \rightarrow(A, \mathrm{Bd} A)$ is a homeomorphism, then $A$ is called a (closed) E-cell in $X, h\left(B_{1}\right)$ is a collared E-cell in $X$, collared by $h$ and $A$ is a tame E-cell if $X=E$ and $h$ extends to a space homeomorphism. On the other hand, if $h:\left(B_{3}-{ }^{\circ} B_{1}\right.$, $\left.S_{3} \cup S_{1}\right) \rightarrow(A, \mathrm{Bd} A)$ is a homeomorphism, $A$ is called a (closed) E-annulus in $X$ and $h\left(S_{2}\right)$ a bicollared $E$-sphere in $X$, bicollared by $h$. The prefix $E$-will be omitted if no confusion is likely.

Received by the editors December 17, 1968. 
The first lemma shows that collared cells and bicollared spheres have a "basis" of collars (compare Lemma 3.1 of [8]).

1.1 Lemma. A collared cell is collared (and a bicollared sphere is bicollared) in every open set containing it.

Proof. It suffices to prove that $B_{1}$ is collared in any open subset $U$ of $E$ containing $B_{1}$. For $1 \leqq t \leqq 2$ and $x \in S_{1}$, set $h(t x)=x+((t-1) / 2) d(x, E-U) x$ where $d(x, E-U)$ is the distance from $x$ to $E-U$. Letting $h(x)=x$ if $x \in B_{1}$ it is clear that $h$ is a homeomorphism which collars $B_{1}$ in $U$. Note that $h$ could also easily be extended to a space homeomorphism supported on $B_{3}-B_{1}$.

1.2 Corollary. If $K$ is a closed set disjoint from a boundary sphere, $S$, of an $E$-cell or E-annulus, $D$, then $S$ is collared in $D-K$ in the sense that the homeomorphism $h$ defining $D$ can be chosen so $h\left(B_{2}-{ }^{\circ} B_{1}\right)$ has $S$ as a boundary component and lies in $D-K$.

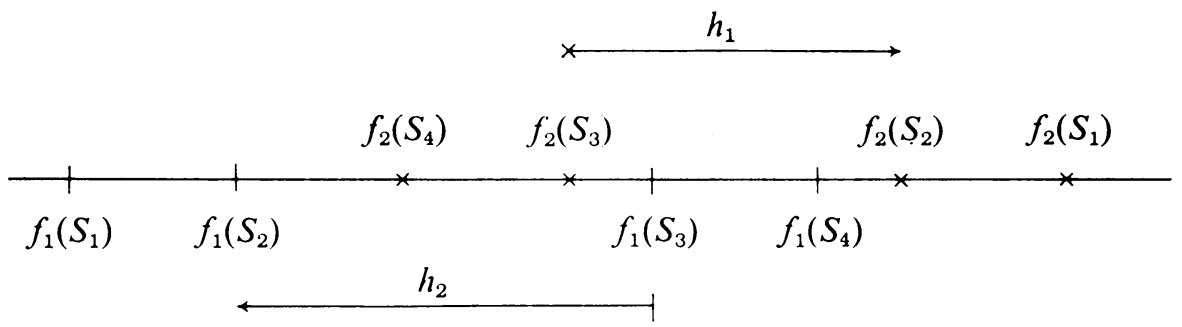

FIGURE 1

Figure 1 symbolically represents the statement and proof of the next result which is an engulfing lemma of a type often found useful in linear spaces.

1.3 Lemma. For $i=1,2$ suppose the homeomorphisms $f_{i}:\left(B_{4}-{ }^{\circ} B_{1}, S_{4} \cup S_{1}\right)$ $\rightarrow\left(A_{i}, \mathrm{Bd} A_{i}\right)$ define $E_{i}$-annuli $A_{i}$ in $X$ such that for $i \neq j=1,2, f_{i}\left(S_{3} \cup S_{4}\right) \subset$ $f_{j}\left({ }^{\circ} B_{3}-B_{2}\right)$ and $f_{i}\left(B_{2}-{ }^{\circ} B_{1}\right) \cap A_{j}=\varnothing$. Then there is a homeomorphism $h$ of $X$ onto itself, supported on $f_{1}\left(B_{3}-B_{1}\right)-f_{2}\left(B_{3}-B_{1}\right)$, with $h f_{2}\left(S_{4}\right) \subset f_{1}\left({ }^{\circ} B_{2}-B_{1}\right)$ and $h\left(A_{2}\right)$ $\supset f_{1}\left(B_{4}-{ }^{\circ} B_{2}\right)$.

Proof. Observe first that the hypotheses of 1.3 imply $f_{j}\left({ }^{\circ} B_{3}-B_{2}\right)$ and $X-$ $f_{j}\left(B_{3}-{ }^{\circ} B_{2}\right)$ are disjoint open subsets of $X$ and thus, in addition, that $f_{i}\left(B_{4}-{ }^{\circ} B_{3}\right)$ $\subset f_{j}\left({ }^{\circ} B_{3}-B_{2}\right)$. To obtain the required homeomorphism, first let $g$ be a radial homeomorphism of $E$ onto itself, supported on $B_{4}-B_{1}$, which maps $S_{3}$ onto $S_{2}$. Then set $h=h_{2}^{-1} h_{1} h_{2}$ where $h_{i}$ is the identity on $X-A_{i}$ and $h_{i} \mid A_{i}=f_{i} g f_{i}^{-1}$. The properties claimed for $h$ in 1.3 are easily verified.

No relation between the linear spaces $E_{1}$ and $E_{2}$ is indicated in 1.3 but it is a consequence of Theorem 1.4 below that they must in fact be homeomorphic under the given conditions. 
1.4 THEOREM. If the normed linear space E contains a collared $E^{\prime}$-cell, then the embedding of the cell extends to a homeomorphism of $E^{\prime}$ onto $E$.

Proof. If $C$ is such a cell, there is a homeomorphism $h=\left(B_{2}^{\prime}, S_{2}^{\prime}\right) \rightarrow(B, \mathrm{Bd} B)$ where $B \subset E$ and $h\left(B_{1}^{\prime}\right)=C$. By inverting $E$ with respect to a ball in $E-h\left(B_{2}^{\prime}\right)$ with a homeomorphism as described in [6], rescaling and translating if necessary, we may assume $h\left(B_{2}^{\prime}\right) \subset{ }^{\circ} B_{k}$ for some $k, B_{2} \subset{ }^{\circ} C$ and that $h\left(\theta^{\prime}\right)=\theta$. Now $B_{1}$ contains $h\left(B_{2 \varepsilon}^{\prime}\right)$ for a sufficiently small $\varepsilon$ and if $r_{i}=2-2^{-i}$, an application of 1.3 with $A_{1}=h\left(B_{r_{1}}^{\prime}-{ }^{\circ} B_{\varepsilon}^{\prime}\right)$ and $A_{2}=B_{k+1}-{ }^{\circ} B_{1}$ obtains a homeomorphism $h_{1}$ of $E$ onto itself, supported on $B_{k+1}-C=B_{k+1}-h\left(B_{r_{0}}^{\prime}\right)$, mapping $h\left(S_{r_{1}}^{\prime}\right)$ into ${ }^{\circ} B_{k+1}-B_{k}$ and such that $h_{1} h\left(B_{r_{1}}^{\prime}\right) \supset B_{k}$. By induction, if for $i<n$ a homeomorphism $h_{i}$ of $E$ onto $E$ is defined, supported on $B_{k+i}-\bar{h}_{i} h\left(B_{r_{i-1}}^{\prime}\right)$ (where $\bar{h}_{i}=h_{i-1} \cdots h_{2} h_{1}, \bar{h}_{1}$ being the identity) mapping $\bar{h}_{i} h\left(S_{r_{i}}^{\prime}\right)$ into ${ }^{\circ} B_{k+i}-B_{k+i-1}$ and such that $\bar{h}_{i+1} h\left(B_{r_{i}}^{\prime}\right) \supset B_{k+i-1}$, then $h_{n}$ is obtained by applying 1.3 with $A_{1}=\bar{h}_{n} h\left(B_{r_{n}}^{\prime}-{ }^{\circ} B_{\varepsilon}^{\prime}\right)$ and $A_{2}=B_{k+n}-{ }^{\circ} B_{n}$ such that $h_{n}$ is supported on $B_{k+n}-\bar{h}_{n} h\left(B_{r_{n-1}}^{\prime}\right)$, maps $\bar{h}_{n} h\left(S_{r_{n}}^{\prime}\right)$ into ${ }^{\circ} B_{k+n}-B_{k+n-1}$ and $\bar{h}_{n+1} h\left(B_{r_{n}}^{\prime}\right) \supset B_{k+n-1}$. Since no point of $\bar{h}_{n+1} h\left(B_{r_{n}}^{\prime}\right)$ is moved by any $h_{i}$ if $i>n$, it follows that $H=\left.\lim _{n-\infty} \bar{h}_{n} h\right|^{\circ} B_{2}^{\prime}$ is a homeomorphism of ${ }^{\circ} B_{2}^{\prime}$ onto $E$ and $H \mid B_{1}^{\prime}=$ $h \mid B_{1}^{\prime}$ from which the conclusion follows.

1.5 Corollary. A collared cell in a normed linear space is point-like (i.e., its complement is homeomorphic to the complement of a point-this corollary is also a consequence of Theorem 3.6 of [8]).

1.6 COROLlaRY. If an open subset of a normed linear space $E^{\prime}$ is embedded in a normed linear space $E$ and the image has nonempty interior, then $E$ and $E^{\prime}$ are homeomorphic.

Proof. Using regularity, a nonempty open subset, $U$, of $E$ can be found whose closure is contained in such an embedding. The inverse of $U$ is an open subset of $E^{\prime}$ and so contains a collared cell whose image in $E$ is also collared. The corollary then follows immediately from 1.4 .

EXAMPLES. (i) Hilbert space, $l_{2}$, is homeomorphic to its hyperplanes and to its unit ball, $B_{1}$ (see [5]). Thus, if $C$ is the cylinder consisting of all points of $l_{2}$ whose projection on the hyperplane $x_{1}=0$ lies in $B_{1}$, there is a homeomorphism, $h$, of $C$ onto $l_{2}$. Since $B_{1} \subset C$ and $S_{1}$ is the boundary of $B_{1}$ relative to $C$, it follows that $h\left(B_{1}\right)$ is an $l_{2}$-cell in $l_{2}$. Since $B_{1}$ separates $C$, it is clear (e.g. from 1.4) that $h\left(B_{1}\right)$ cannot be collared in $l_{2}$.

(ii) Since $l_{2}$ is also homeomorphic to its unit sphere [5], to $l_{2} \times(0,1]$ and to $l_{2} \times T$, where $T$ is a triod [1], then $B_{1}$ is a "sphere" in $l_{2}$ which does not separate, $B_{1}-\theta$ is a "sphere" in $l_{2}$ which separates $l_{2}$ into two components but is not the common boundary, and $l_{2} \times p$ ( $p$ being the triod point of $T$ ) is a "sphere" in the copy, $l_{2} \times T$, of Hilbert space whose complement has three components. The next result, a sort of "Jordan Curve Theorem" for infinite-dimensional normed linear spaces, shows that none of the above can hold true for bicollared spheres. 
1.7 THEOREM. A bicollared sphere in a normed linear space $E$ separates $E$ into two components and is the boundary of each.

Proof. If $h$ bicollars a sphere $S$ in $E$ then $S$ separates $h\left(B_{3}-{ }^{\circ} B_{1}\right)$ into two components $A, B$ and is in the interior of their union so that a ray from any point $p$ of $E-h\left(B_{3}-{ }^{\circ} B_{1}\right)$, which meets $S$ has the property that the open segment $(p, q)$, where $q$ is the first point of $S$ on the ray, contains a point of $A$ or $B$. Since $A$ and $B$ are connected, it follows that $E-S$ has at most two components and $S$ is the boundary of each. Suppose $S$ does not separate $E$ and $p \in S$. A sufficiently small ball centered at $p$ lies in $\mathrm{cl}(A \cup B)$ and must contain points $a \in A, b \in B$ which lie, together with $a p$ and $b p$, on a polygonal simple closed curve $L$ with vertices in general position, with subarc $a p b$ contained in $\mathrm{cl}(A \cup B)$ and with $L-a p b$ contained in $E-S$. Then the component of $\operatorname{cl}(A \cup B) \cap L$ containing $p$ has endpoints $a^{\prime} \in A, b^{\prime} \in B$. Also, the join of $p$ to $\operatorname{cl}(L-a p b)$ is a disk $D$. Let $C$ be the component of $\operatorname{cl}(A \cup B) \cap D$ containing $p, K$ the union of $C$ and all components of $D-C$ with closures disjoint from $L$ ( $K$ is $C$ with its "holes" filled in) and $K^{\prime}$ the closure of the union of $L-a^{\prime} p b^{\prime}$ and the remaining components of $D-C$. Then since $D=K \cup K^{\prime}$ is unicoherent and $K, K^{\prime}$ are continua, it follows that $K \cap K^{\prime}$ is connected, contains $a^{\prime}$ and $b^{\prime}$ and lies in $A \cup B$, contradicting the definition of $A$ and $B$. Thus $S$ separates $E$ and the proof is complete.

1.8 Lemma. If $C$ is an E-cell in $X$ and $A$ is an E-annulus with boundary spheres $S^{\prime} \subset{ }^{\circ} C$ and $S \subset X-{ }^{\circ} C$, then $A \cup C$ is an E-cell in $X$ with boundary $S$.

Proof. We may assume without loss of generality the existence of a collared ball $B$ in $C-A$ "concentric" with $C$ (i.e. the image of $B_{\varepsilon}$ for some $\varepsilon>0$ under the homeomorphism which defines $C$ ). Because of 1.2 we may also assume $A \cap{ }^{\circ} C$ contains a collar $K$ of $S^{\prime}$ and that $C$ is disjoint from $S$ (i.e. we may replace $C$ by a slightly "smaller" cell) and contains a collar of its boundary disjoint from $K$. It then follows, again by 1.2 , that $A-C$ contains a collar of $S$. Thus 1.3 can be applied with $C-{ }^{\circ} B$ and $A$ in place of $A_{1}$ and $A_{2}$ respectively, to get a homeomorphism mapping $S^{\prime}$ into the collar of $B$ and supported on $K \cup(C-A-B)$. This procedure can be iterated as in the proof of 1.4 to obtain a homeomorphism of $A-S^{\prime}$ onto $A \cup C-p$ where $p$ is the "center" of $C$. Since $C$ is homeomorphic to $C-p$ keeping the boundary of $C$ fixed [6], it follows that $A \cup C$ is an $E$-cell in $X$ with boundary $S$.

1.9 THEOREM. If $X$ is the union of the interiors of two E-cells in $X$ then $X$ is homeomorphic to $E$.

Proof. If $X={ }^{\circ} C \cup{ }^{\circ} C^{\prime}$ where $C, C^{\prime}$ are $E$-cells in $X$, then there is a ball $B \subset X-C$ "concentric" with $C^{\prime}$ (in the sense described in the proof of 1.8) such that $C$ and $A=C^{\prime}-{ }^{\circ} B$ satisfy the hypotheses of 1.8 . Then $A \cup C$ is an $E$-cell in $X$ with boundary equal to that of $B$ and $X=A \cup C \cup B$ is homeomorphic to $E$. 
1.10 COROLLARY. If $X$ is the union of two E-cells in $X$, one of which is collared, then $X$ is homeomorphic to $E$.

Proof. The union of the collared cell with its collar is an $E$-cell in $X$ whose interior together with the interior of the other cell cover $X$ so the corollary follows immediately from the theorem.

The corollary may be true without the condition that one of the cells be collared but this is still an open question. A finite-dimensional version of 1.9 has been observed by several persons, e.g. in [12] and [13], as an application of Morton Brown's Generalized Schoenflies Theorem [3]. It is a consequence of the next theorem that, under the conditions of 1.9, the intersection of the open $E$-cells is also homeomorphic to $E$. Thus these results relate to questions recently raised by R. D. Anderson and R. H. Bing (questions 17 and 19 of [2]) as to whether, given two sets homeomorphic to Hilbert space and both open (as in 1.9) or both closed (as in 1.10) in their union, either their union or intersection being homeomorphic to Hilbert space makes the other one homeomorphic to Hilbert space. In 1.9 and 1.10 a stronger condition is imposed on the two sets but the hypothesis on the intersection becomes (by virtue of 1.11) part of the conclusion in the open case. Furthermore, both apply to any normed linear space. Further results along this line are collected in the first corollary to the infinite-dimensional Schoenflies theorem which is proved next.

1.11 THEOREM. A bicollared sphere $S$ in a normed linear space $E$ splits $E$ (i.e., $E-S$ has two components whose closures are E-cells).

Proof. By a change of origin, scale and an inversion we may assume $S$ to be bicollared in ${ }^{\circ} B_{1}$, by a homeomorphism $k$ such that $E-k\left(B_{3}-{ }^{\circ} B_{1}\right) \subset{ }^{\circ} B_{1}$. Adjoining a point $\omega$ to $E$ with neighborhoods of the form $\omega \cup U$ where $E-U$ is closed and bounded in $E$ gives a space $\widetilde{E}$ which is homeomorphic to $E$ (called the one point cobounded extension of $E$ by R. A. McCoy [8]). By 1.7, $E-k\left(B_{3}-{ }^{\circ} B_{1}\right)$ has two components whose closures will be denoted by $P$ and $Q$ where $P \supset k\left(S_{1}\right)$. A map $f$ of $E$ onto $\tilde{E}$ is defined by setting $f(P)=\theta, f(Q)=\omega$ and

$$
f(x)=\frac{\left|k^{-1}(x)\right|-1}{3-\left|k^{-1}(x)\right|} k^{-1}(x) \text { for } x \in E-(P \cup Q) .
$$

Clearly $f \mid E-(P \cup Q)$ is a homeomorphism and $f$ is continuous on $P$ (likewise $Q$ ) since $f(P)=\theta$ has a neighborhood basis of concentric open cells the inverse of each of which is the complement of the union of the closed set $Q$ and a closed annulus in $k\left(B_{3}-{ }^{\circ} B_{1}\right)$. Since $E-{ }^{\circ} B_{2}$ is a collared cell in $E-(P \cup Q), 1.4$ implies $f\left(E-{ }^{\circ} B_{2}\right)$ and hence also $f\left(B_{2}\right)$ is a tame cell in $\tilde{E}$ and the latter cell contains both $\theta$ and $\omega$. Choose $\varepsilon>0$ so that $B_{2 \varepsilon} \subset f\left({ }^{\circ} B_{2}\right)$ and apply 1.3 to define a homeomorphism $g$ of $\tilde{E}$ onto itself such that $g f\left(B_{2}\right) \subset B_{2 \varepsilon}$ and $g \mid B_{\varepsilon}$ is the identity. Define a map $G$ of $B_{2}$ into itself by letting $G \mid f^{-1}\left(B_{\varepsilon}\right)$ be the identity and $G \mid B_{2}-f^{-1}\left(B_{\varepsilon}\right)=$ $f^{-1} g f \mid B_{2}-f^{-1}\left(B_{\varepsilon}\right)$. The map $G$ has one inverse set, $Q$, and is a homeomorphism on $B_{2}-Q$. Choose $\delta>0$ so that (denoting by $B_{t}(p)$ the ball of radius $t$ centered at $p$ ) 
$B_{2 \delta}(G(Q)) \subset G k\left(B_{3}-B_{2}\right)=G f^{-1}\left(\tilde{E}-B_{2}\right)$ and apply 1.3 once more (or a simple radial description) to get a homeomorphism $h$ of $B_{2}$ into $B_{2 \delta}(G(Q))$ which is the identity on $B_{\delta}(G(Q))$. Choose $\gamma>0$ so that $G k\left(B_{3}-B_{3-\gamma}\right) \subset B_{\delta}(G(Q))$ and define $H$ on $B_{2}$ as the identity on $k\left(B_{3}-B_{3-\gamma}\right) \cup Q=U$ and $H \mid B_{2}-U=G^{-1} h G$. Then $H \mid B_{1}$ is a homeomorphism of $B_{1}$ onto a collared cell $C$ containing $Q$ such that $C$ and $A=k\left(B_{3}-{ }^{\circ} B_{2}\right)$ satisfy the hypotheses of 1.8 (where $\left.k\left(S_{3}\right)=S^{\prime}\right)$ so that $A \cup C$ $=k\left(B_{3}-{ }^{\circ} B_{2}\right) \cup Q$ is a cell in $E$ with boundary $S$. Similarly, $k\left(B_{2}-{ }^{\circ} B_{1}\right) \cup P$ is a cell with boundary $S$ and the proof is complete.

1.12 CoRollary. If $X=H \cup K$ where both $H$ and $K$ are open sets homeomorphic to a normed linear space $E$, then under any of the following conditions, $X$ is homeomorphic to $E$ :

(i) $H-K$ and $K-H$ are separated by a bicollared sphere in $X$.

(ii) $H-K$ or $K-H$ has a (closed) neighborhood which is an E-cell in $X$.

(iii) $H-K$ or $K-H$ has a basis of neighborhoods which are E-cells in $H$ or $K$, respectively.

Proof. Observe first that an $E$-cell in $H$ or $K$ need not be an $E$-cell in $X$ (indeed, its boundary in $X$ may not even be connected). However, since $X$ is normal, $H-K$ and $K-H$ have neighborhoods with disjoint closures and an $E$-cell in $H$ or $K$ contained in one of these neighborhoods will be an $E$-cell in $X$, so (iii) is sufficient if (ii) is. By 1.2, condition (ii) implies $H-K$ (or $K-H$ as the case may be) is contained in a collared $E$-cell in $X$, call it $C$. The boundary, $S$, of $C$ is a bicollared sphere in $X$ contained in the open set $K$ and hence bicollared in $K$ according to 1.1. By $1.11, S$ splits $K$ so that $X$ is the union of $C$ and one of the components of $K-S$ whose closure is an $E$-cell in $X$ as well as in $K$. Thus $X$ is homeomorphic to $E$. The sufficiency of (i) follows similarly since a bicollared sphere in $X$ separating $H-K$ and $K-H$ will be bicollared in both $H$ and $K$. Hence $X$ is the union of two $E$-cells, one in $H$ and one in $K$, with the sphere as their common boundary.

Condition (iii) is strictly intermediate between what McCoy calls cellularity and strong cellularity of $H-K$ or $K-H$. Furthermore, the condition in problem 17 of [2] that $H \cap K$ be homeomorphic to $E$ is equivalent to requiring both $H-K$ and $K-H$ to be point-like in $H$ and $K$, respectively (as defined in 1.5). Theorem 3.6 of [8] shows that cellularity implies point-like. It seems likely that they are equivalent but this remains an open question.

1.13 CoRollary. A normed linear space, $E$, is homeomorphic to its unit ball, $B_{1}$, if and only if it is homeomorphic to its (closed) annulus, $B_{3}-{ }^{\circ} B_{1}$.

Proof. Suppose $E$ is homeomorphic to $B_{1}$. Since both $B_{3}-B_{1}$ and ${ }^{\circ} B_{3}-{ }^{\circ} B_{1}$ are homeomorphic to $B_{1}$ and since $S_{2}$ is bicollared in each, it follows from 1.11 (applied twice) that $B_{3}-{ }^{\circ} B_{1}$ is the union of two $E$-cells with common boundary, $S_{2}$. Thus $E$ is homeomorphic to the annulus $B_{3}-{ }^{\circ} B_{1}$. On the other hand, if $E$ is homeomorphic to $B_{3}-{ }^{\circ} B_{1}, S_{2}$ is bicollared in $B_{3}-{ }^{\circ} B_{1}$ and so 1.11 implies that $B_{1}$ is homeomorphic to $B_{2}-{ }^{\circ} B_{1}$ and therefore to $E$. 
Corollary 1.13 appears to have implications in the classification of convex sets in normed linear spaces which the author expects to develop in a later paper. A similar application of 1.11 provides an infinite-dimensional annulus theorem:

1.14 Corollary. If $C$ is a tame E-cell in the interior of an E-cell $C^{\prime}$, and if $E$ is homeomorphic to its unit ball, then $C^{\prime}-{ }^{\circ} \mathrm{C}$ is homeomorphic to an annulus.

Proof. By 1.2, the boundary of $C^{\prime}$ is collared in $C^{\prime}-C$ and so ${ }^{\circ} C^{\prime}-C$ contains a bicollared sphere $S$ which separates $C$ from the boundary of $C^{\prime}$. By McCoy's half-open annulus theorem (Theorem 6.2 of [8]), both $C^{\prime}-C$ and ${ }^{\circ} C^{\prime}-{ }^{\circ} C$ are homeomorphic to $B_{1}$, hence to $E$. As in 1.13 , Theorem 1.11 implies $C^{\prime}-{ }^{\circ} C$ is the union of two $E$-cells with common boundary and is homeomorphic to $E$. Since $E$ is homeomorphic to $B_{1}$, it follows that $C^{\prime}-{ }^{\circ} C$ is homeomorphic to $B_{3}-{ }^{\circ} B_{1}$, an annulus.

As McCoy points out in [8], it remains to be shown whether or not the homeomorphism between $C^{\prime}-{ }^{\circ} C$ and $B_{3}-{ }^{\circ} B_{1}$ can be chosen to map the boundaries of $C$ and $C^{\prime}$ onto $S_{3}$ and $S_{1}$, respectively (that is, whether or not $C^{\prime}-{ }^{\circ} C$ is an E-annulus).

\section{REFERENCES}

1. R. D. Anderson, Topological properties of the Hilbert cube and the infinite product of open intervals, Trans. Amer. Math. Soc. 126 (1967), 200-216. MR 34 \#5045.

2. R. D. Anderson and R. H. Bing, A complete elementary proof that Hilbert space is homeomorphic to the countable infinite product of lines, Bull. Amer. Math. Soc. 74 (1968), 771-792. MR 37 \#5847.

3. M. Brown, A proof of the generalized Schoenflies theorem, Bull. Amer. Math. Soc. 66 (1960), 74-76. MR 22 \#8470b.

4. M. L. Curtis and E. C. Zeeman, On the polyhedral Schoenflies theorem, Proc. Amer. Math. Soc. 11 (1960), 888-889. MR 22 \#9974.

5. V. L. Klee, Convex bodies and periodic homeomorphisms in Hilbert space, Trans. Amer. Math. Soc. 74 (1953), 10-43. MR 14, 989.

6. - A note on topological properties of normed linear spaces, Proc. Amer. Math. Soc. 7 (1956), 673-674. MR 17, 1227.

7. B. Mazur, On embeddings of spheres, Bull. Amer. Math. Soc. 65 (1959), 59-65. MR 22 \#8469.

8. R. A. McCoy, Cells and cellularity in infinite-dimensional normed linear spaces, (to appear).

9. M. Morse, A reduction of the Schoenflies extension problem, Bull. Amer. Math. Soc. 66 (1960), 113-115. MR 22 \#8470a.

10. M. H. A. Newman, On the division of Euclidean $n$-space by topological $(n-1)$-spheres, Proc. Roy. Soc. London Ser. A 257 (1960), 1-12. MR 23 A633.

11. P. M. Rice, The Hauptvermutung and the polyhedral Schoenfies theorem, Bull. Amer. Math. Soc. 71 (1965), 521-522. MR 31 \# 2728.

12. R. H. Rosen, Stellar neighborhoods in polyhedral manifolds, Proc. Amer. Math. Soc. 14 (1963), 401-406. MR 27 \#768.

13. J. R. Stallings, Polyhedral homotopy-spheres, Bull. Amer. Math. Soc. 66 (1960), 485-488. MR 23 \#A2214.

Iowa State University of Science and Technology, AMES, IOWA 